\title{
Context and conditioning: A place for space
}

\author{
LYNN NADEL and JEFFREY WILLNER \\ Dalhousie University, Halifax, Nova Scotia B3H 4H8, Canada
}

\begin{abstract}
Notions about the influence of environmental context on behavior are briefly reviewed and criticized. In contrast to the traditional view that a context is merely a compound CS, to be treated in learning theory in much the same way as simple CSs, we propose that contexts are superordinate to such CSs. Within this hierarchical relation, a context both contains and predicts CSs. This approach to environmental context derives from cognitive map theory (O'Keefe \& Nadel, 1978), and predictions based on that theory and the present extension are offered for several conditioning paradigms.
\end{abstract}

In parenthesis it may be remarked that this fact that supports, and not merely stimuli, are needed for the actual going off of any act, and are expected by such an act, is a feature about behavior which orthodox psychologies, both stimulus-response psychologies and mentalisms, seem hitherto to have overlooked (Tolman, 1932, p. 85).

The "supports" of which Tolman spoke are the contexts of today, and if it is not nearly so true that orthodox psychologies have overlooked contexts, neither have they particularly illuminated them. Traditional preference, at least for English-speaking psychologists, has been to consider environmental supports, or contexts, as merely additional stimuli about which the organism can learn and to which associations can be formed in the overall competition for associative strength. In contrast to this egalitarian view, we will argue that environmental context is quite different from stimuli such as a buzzer, tone, or flashing light. Put most succinctly, we will assert that contexts contain and predict, rather than simply compete with, such stimuli. This hierarchical relation between context and its contents will be shown to have several interesting implications for the analysis of context effects in conditioning. Further, it will connect the study of context with that of spatial mapping and place learning. This drawing together of contexts, spatial maps, and learning about places provides a foundation for a cognitive theory of

This paper is the result of prolonged collaboration aimed at developing an approach to context couched within the framework of the O'Keefe/Nadel (1978) cognitive map theory. It was a truly joint effort. We thank W. K. Honig, W. J. Jacobs, V. M. LoLordo, A. Randich, and other members of the animal learning group at Dalhousie University for their stimulating conversations and company, which contributed to all the good ideas herein, but (of course) none of the bad. We also thank J. O'Keefe and A. H. Black for early discussion of some of these issues. Our work on this paper was supported by grants from NSERC (L. Nadel), NATO (A. H. Black), NSERC (W. K. Honig), and Dalhousie University. L. Nadel's current address: 588 Estrelita Drive, Vista, California 92083. Reprints may be obtained from either author. animal learning that makes contact with current physiological thinking (cf. O'Keefe \& Nadel, 1978).

Our argument will comprise three stages. First, we will briefly define context and consider its treatment in traditional learning theory. Second, our discussion of the shortcomings of the traditional approach to context will set the stage for a sketch of a new perspective on context, one which draws heavily on recent research with the neural substrate of spatial mapping, the hippocampus. Finally, this approach will be applied to several conditioning situations to indicate its strengths (and weaknesses) and to suggest potentially fruitful areas for future research.

\section{The Nature of Context}

The term "context" has been used in such a bewildering variety of ways that one is tempted to conclude that it defies accurate definition. Funk and Wagnall's provides two related senses, one applying to verbal contexts, the other to nonverbal ones. Both convey the notion of something that surrounds and influences. This "something,"' however, can be any of innumerable environments or circumstances that surround/influence. For example, environments of interest to the psycholinguist include such things as the set of speech sounds in which a particular sound is embedded, or the words adjacent to the target word in a list, or perhaps the sentences of a paragraph within which these speech sounds or words occur. For the anthropologist, it could be the particular socioeconomic situation, or possibly the cultural or political circumstances, within which specific patterns of behavior are manifested.

It seems clear that the list of possible contexts of interest must include: spatial, temporal, associative, cultural, economic, political, social, to name a few. Each discipline chooses the set of contexts, or frames of reference, it wishes to study. It is a curious historical fact that most psychologists have paid scant attention to environmental context, even though this should be a "context" of more than passing interest to a science of behavior. 
This is not to say that environmental context has been totally ignored. Hull (1943) and Skinner (1938) both recognized the importance of context, but neither pursued the problem. Skinner, for example, noted that:

The stimulus to be conditioned must be presented against a background of stimulation arising from the situation .... The power to elicit the response is acquired not only by the precise combination of stimuli present at the moment but also by the component parts. Consequently the dog may salivate when put into the stand again or when the tone is sounded in entirely different surroundings. This triple conditioning cannot wholly be avoided (Skinner, 1938, p. 177).

He then went on to suggest ways to avoid conditioning to the background, but concluded that "in spite of them some induction to the experimental situation occurs"' (Skinner, 1938, p. 177). Much as the operant chamber finessed the interesting questions of the spatial aspects of behavior, Skinner sought a way around this question of background by setting up a discrimination which eliminates conditioning to the context (or tries to) by presenting the context without the discriminative stimulus and without reward. Today, we call this an "adaptation period" and neglect the interesting learning that occurs before our experiments even begin.

Hull (1943) set his discussion of this issue in the context of an analysis of the factors controlling the selectivity of associations entered into by the various stimuli available in the experimental task:

\footnotetext{
Recognition of the variability in the increments of habit strength acquired by the several stimulus components of a conditioning situation at once raises the question of the principles according to which the different magnitudes of habit-strength loadings arise .... Stimuli which have continued to act on a receptor without change for some time have a greatly diminished capacity for acquiring habit loadings. Partly for this reason it is probable that static, i.e., unchanging, elements or aggregates in a conditioned stimulus situation are considerably less potent in the acquisition of habit-strength loadings than are the more dynamic, i.e., changing elements or aggregates. This probably is why investigators so frequently neglect to take into consideration the static or constant elements of conditioned stimulus situations, sometimes with unfortunate results (Hull, 1943, pp. 207-208).
}

Here, Hull foreshadowed an approach taken by some latter-day Pavlovians (e.g., Asratyan, 1965), who have laid great emphasis on the differences between static ("tonic") and dynamic ("phasic") elements in the conditioning situation. Unfortunately, Hull did not take this issue up, and Asratyan's program has not yet been applied to a consideration of environmental context (cf. Kimmel \& Ray, 1978).

Hull's learning theory was supplanted during the 1950s by several less ambitious models; statistical learning theory (cf. Estes, 1959) was one of the more important of these approaches. Estes assumed that all stimulus elements were conditionable but that only some portion of these were perceived, or attended to, on any given trial. Only these would acquire habit strength on that trial. No special status was assigned to any form of stimulus, static or dynamic; all stimuli were equally likely to be available for conditioning.

This "all stimuli are created equal" position, a significant change from Hull's more sophisticated, if unrealized, vision, mirrored the position taken by Restle (1957) in his "resolution" of the "place-vs.response" learning controversy. Restle concluded that both forms of learning exist, indeed that they are of the same sort, only that they rely upon different sources for their stimuli. Place learning was held to require extramaze stimuli; response learning required interoceptively generated stimuli. For Restle, all learning is the same, a position directly opposed to Tolman's (1949) claim that there are different kinds of learning. The parallel between Estes' and Restle's contributions seems clear now. Both the amalgamation of environmental context to random stimulus elements and that of place learning to distal stimulus learning contributed heavily to the lack of interest in these topics within the past few decades, at least in the West.

Such was not the case in Eastern Europe. Pavlov (1927) noted that environmental contexts were important parts of what the animal learns in a standard classical conditioning procedure. Shifting some feature of the test situation, or moving the animal to another room, would generally cause a breakdown in performance. Asratyan (1965) studied extensively, through his analysis of the differences between tonic and phasic stimuli, the ability of animals to use contexts as discriminative stimuli and came to the conclusion that environmental context is taken by the animal to be all that is static in the situation, the so-called tonic factor. Konorski (1967) also recognized the importance of distinguishing between tonic and phasic stimuli. According to Konorski, a "preparatory" conditioned response (CR) becomes conditioned to the experimental situation, often after only a single trial. These preparatory CRs are important in that they provide the basis for the subsequent emergence of "consummatory" CRs to the phasic conditioned stimulus (CS). He also asserted that associations between phasic CSs and the static physical context in which they were presented were formed during conditioning, anticipating the approach recently taken by Wagner $(1978,1979)$. Thus, both Asratyan and Konorski suggested important qualitative differences between the static stimuli comprising the environmental context and the phasic stimuli presented in those contexts.

An even more advanced, and determined, con- 
textualist position was developed by Beritoff (1965):

At the very beginning of our studies of conditioned defensive reflexes, we came to the conclusion that the experimental situation, namely, the room itself in which the experiment was conducted, is significant in eliciting a dog's reaction to a conditioned stimulus (Beritoff, 1965, pp. 62-63).

Beritoff assumed that the internal representation of the spatial environment, the dog's "image," could be retrieved and would then play a directing role-he termed this the setting effect. This apparently sensible view of context had little impact in the West, possibly because of its insistence upon internal representations of the sort espoused by Tolman but rejected by most mainstream psychologists.

The past decade has witnessed a renewal of interest in developing theoretical accounts of the conditioning process. Central to this resurgence were the demonstrations of provocative phenomena such as contingency learning (Rescorla, 1968, 1969a) and blocking (Kamin, 1969) which challenged traditional conceptions of conditioning. A general statement that encompasses these (and other) findings is that conditioning to a reference stimulus (hereafter referred to as a nominal CS) is a function not only of the history of reinforcement of that CS (Hull's position), but also depends on the conditioning history of other stimuli in the experimental situation. Two popular theories that embody this notion are those of Mackintosh (1975) and Rescorla and Wagner (1972), although they do so by means of fundamentally different assumptions. In the broadest sense, both theories are about the way in which "context" influences conditioning, but by this they mean the context provided by one nominal CS for another as well as the context provided by the environment as a whole. Both forms of context are treated identically in these models, neither enjoying any special status. Environmental context is treated as simply another CS (admittedly a compound one) which can be associated with an unconditioned stimulus (US). It becomes a determinant of conditioning insofar as it interacts with other stimuli in the situation, just as other CSs interact with each other. Both models are capable of integrating and explaining the results of a variety of experiments; however, there are good reasons to question the central assumptions of each.

Like other preceding models, the Rescorla-Wagner model assumes that there is a fixed amount of "associative strength" which the US can support. Learning occurs whenever there is a discrepancy between the associative strength which the US can support and that already conditioned to the aggregate of cues present on that trial. Depending on the sign of this discrepancy (positive or negative), each of the cues will acquire either excitatory or inhibitory associative strength, according to its "salience" (a fixed, learning rate parameter unique to each stimulus). Since the model postulates that there is a limited amount of associative strength that must be shared among CSs, any conditioning to one CS necessarily limits the associative strength available to be conditioned to the other CSs present on that trial. This "competition" assumption allows the model to account for a variety of phenomena in compound conditioning, like blocking (Kamin, 1969). Blocking refers to the finding that prior conditioning of one CS will attenuate conditioning of a second CS when they are subsequently reinforced in compound. The model accounts for this effect by assuming that the prior conditioning of the first CS preempts the associative strength which would otherwise accrue to the second CS during compound conditioning.

The role of environmental context within the model is exemplified by its treatment of the "contingency" experiments of Rescorla (1968, 1969a). In fear conditioning experiments with rats, Rescorla (1968) found that excitatory conditioning of a nominal CS depended not only on the probability of shock in the presence of the CS, but also on the probability of shock in the absence of the CS. He observed that the CS became a conditioned excitor only when shock was more likely in the presence of the CS than in its absence. For a given probability of shock in the presence of the CS, conditioning was a decreasing function of the probability of shock in the absence of the CS; as shocks became more frequent in the absence of the CS, fear of the nominal CS decreased. Furthermore, he observed essentially no conditioning of the nominal CS when shocks were equally likely to occur in the presence and absence of the nominal CS. ${ }^{1}$ Rescorla (1969a) extended these findings to the case of conditioned inhibition by showing (1) that a CS became a conditioned inhibitor when the US was more likely to occur in the absence of the CS than in its presence, and (2) that the magnitude of this inhibitory conditioning was directly related to the probability of the US in the absence of the CS.

At first blush, such data suggest that animals are sophisticated mathematicians, integrating events over time, calculating probabilities, and acting according to this information. By assuming that the context can be treated as any nominal CS, the Rescorla-Wagner model provides what appears to be a straightforward, mechanistic account of the data. Wagner and Rescorla (1972) note that:

Whenever the animal is in the conditioning chamber, it is subjected to a variety of stimuli in many different modalities, so-called background stimuli. One way of conceptualizing the presentation of an explicit CS is as transforming the stimulation which the subject receives from background stimuli alone into a stimulus com- 
pound consisting of the background plus the explicit CS. If we identify the background stimulus as A and the CS as $\mathrm{X}$, presentation of the CS transforms the stimulation from $A$ into $A X(p .315)$.

We have already seen that the model can be applied to predicting conditioning to one stimulus in a reinforced compound as a function of the separate treatment of the other stimulus. Conditioning to a context can influence learning about a nominal CS in two related ways: When the US has been presented in that context, associative strength will accrue to the context, leaving less for the CS when it is subsequently paired with the US in that context. Should the CS be presented without the US, however, excitatory conditioning of the context may turn the CS into a conditioned inhibitor.

The notion of context conditioning has also been utilized by proponents of this approach to account for the observation that preconditioning experience with the US typically retards subsequent conditioning of a nominal CS paired with that US (e.g., Tomie, 1976; Hinson \& Siegel, Note 1). These associative accounts of the "US preexposure effect" (Randlich \& LoLordo, 1979) generally presume that the context becomes conditioned during the preexposure phase. During subsequent conditioning, the presence of these strongly conditioned contextual cues interferes with conditioning to the nominal CS by preempting associative strength which would otherwise have been available to be conditioned to the CS. The role of context in this case is similar to that of the pretrained cue in blocking.

Context, then, is simply another CS which can be conditioned, and its role in learning is to be understood in terms of competition among cues for a limited amount of associative strength. Are "contextual cues" conditioned in the manner postulated by the Rescorla-Wagner model? Confirmatory evidence on this question has been provided by Dweck and Wagner (1970) and Kremer (1974) in studies on the truly random control procedure, and by Odling-Smee (1975a). These studies have shown that environmental context can be conditioned, and they provide some support for the model's competition hypothesis. According to the model, signaling the US should reduce context conditioning, in that the CS should come to "overshadow" the context. This prediction was also confirmed in that these studies found an inverse relation between contextual fear and the number of signaled USs.

Despite its success in predicting the outcomes of a variety of classical conditioning experiments, the influence of the Rescorla-Wagner model has waned in recent years. This decline can be traced to two different sources. First, the model does not address the phenomenon of latent inhibition (Lubow, 1973), the finding that nonreinforced exposure to a stimulus typically retards subsequent conditioning of that stimulus. Most theorists would agree that latent inhibition represents some type of learning process and that an adequate theory of learning must provide an explanation for it. More important than this apparent error of omission, though, are data contradicting the model's assumption that there is a limited amount of associative strength to be shared among the CSs present on a conditioning trial.

These data come from experiments on "unblocking." Unblocking refers to the fact that there are a variety of manipulations that will result in conditioning to the added cue in the blocking paradigm, such as increasing the intensity of shock or adding an additional shock at the start of compound training (Kamin, 1969). According to the Rescorla-Wagner model, blocking occurs because the pretrained CS has preempted all the associative strength that the US can support, as noted above. The only manipulations that should produce unblocking, then, are those that can be construed as increasing the magnitude of reinforcement.

Dickinson, Hall, and Mackintosh (1976) performed an unblocking study which tested this prediction. During the pretraining phase of their experiment, the rats received a CS paired with either a single shock or a double shock. Half the rats from each condition then received compound conditioning, with the compound signaling a single shock, while the compound signaled a double shock for the remaining rats. They found that conditioning to the added cue was blocked when the rats received either a single or a double shock throughout training. However, either the addition of an unexpected shock or the omission of an expected one during compound training produced unblocking. The Rescorla-Wagner model has serious difficulties accounting for how unblocking can be caused by the omission of an expected US. In place of the assumption that there is a fixed amount of associative strength to be shared among elements of fixed salience, Mackintosh's (1975) theory of attention asserts that the associability of stimuli with reinforcement (their salience) changes as a function of the animal's experience with those stimuli. Mackintosh assumes that the salience of a CS increases when that CS is the best predictor of the outcome of a trial, and decreases when it isn't. Interactions among CSs are thus to be understood in terms of changes in the salience of each CS.

Context becomes important in Mackintosh's treatment of latent inhibition and "learned irrelevance" (Mackintosh, 1973). We have already mentioned latent inhibition. Learned irrelevance is similar to latent inhibition, but refers instead to the fact that the nominal CS from a truly random control procedure is subsequently retarded in acquiring either excitatory or inhibitory properties (Baker \& Mackintosh, 1977); the animal learns that the stimulus 
is "irrelevant." In his discussion of these phenomena, Mackintosh noted that:

A CS [can] be regarded as forming a compound with a set of background stimuli, C. The crucial point, then, is that the presentation of AC (CS plus background) predicts no change from the probability of reinforcement or nonreinforcement predicted by $\mathrm{C}$ alone (Mackintosh, 1975, p. 289).

Two things are apparent here. First, Mackintosh's conceptualization of context is virtually identical to that of Rescorla and Wagner (1972); context is the same as any other CS. Second, context will enter into the same sorts of interactions among CSs as those we have already described. The only change here is that context exerts its influence on other CSs as one of the variables which determine the salience of those CSs.

The notion that the associability of a CS changes as a function of the organism's experience has much to recommend it. One might wonder, though, whether the salience of a CS changes in the manner postulated by Mackintosh. Hall and Pearce (1979) have obtained data which directly contradict this notion and suggest that if experience does alter the associability of stimuli, its effects are generally decremental.

These reasons to doubt the central assumptions of both the Rescorla-Wagner model and Mackintosh's theory of attention have encouraged us to explore other avenues in our search for a comprehensive theory of learning. We would suggest that past difficulties in formulating an adequate theory stem partly from an inappropriate characterization of the role of environmental context in learning. In the next section, we sketch a new approach to context and provide some discussion of its roots in prior work on cognitive maps.

\section{Rethinking Context}

What has made the analysis of context so confusing is the apparent paradox that a given "context" is both made up of and contains the same sort of things. For example, when we speak of the context of an operant test chamber, we are referring to the various background stimuli to be consistently found there-the lever, the walls, the grid floors, etc. Thought of in this simplified fashion, it is not hard to see how such stimuli, and the context they define, were taken as equivalent to other stimuli such as tones and lights. But the context, the test chamber itself, although composed of these stimuli, also contains them, and it is in this sense that contexts both surround and influence. Consider the fate of the family piano. For purposes of describing the piano, one says that it is in the context of the living room, which one takes as being composed of chairs, sofa, tables, bookcases, pillows, etc. For purposes of describing the sofa, one says that it is in the living room, com- posed of chairs, piano, pillows, tables, etc. Thus, what is background can also be foreground, depending upon one's perspective or purpose. In this way, the stimuli comprising contexts both define (when in the background) and are contained by (when in the foreground) this framework. We will see that this ambiguous status of contextual "cues" results from the way that contexts are represented in the organism's nervous system. It seems clear from this analysis, however, that the elements that move in and out of the foreground are not at the same level as the wholes, or contexts, which they sometimes compose. The relationship between contexts and nominal cues should be seen as hierarchical, rather than parallel. Thus, it is surely incorrect to speak of direct competition between a context and its contents as previous models have done. The foregoing indicates that context is something quite different from nominal CSs or specific stimuli, but we need to look in other directions for clues as to what it might be.

Why does a certain set of stimuli in a given situation come to have this special status of defining context? What exactly is context for the animal, and how is it learned about and internally represented? Current data suggest that the answer goes something like this: when put into a new environment, animals will, after some initial caution, explore the whole of it. As a result of this exploration, the animal learns about the situation; the outcome of this learning process is the construction within the brain of a "map" of the environment. This internal map represents objects and suchlike standing in appropriate spatial relations to one another. Once an element in the situation has been noticed and explored, it will be represented in its appropriate location in the map; such maps are the embodiment of environmental context.

Although organisms seem not to be noticing all the familiar things around them (ignoring background stimuli), as soon as anything changes they react with orienting and exploration, indicating that they are, indeed, continually tracking these familiar features. The exploration triggered by novelty detection serves to revise and update the map of the environment; in this way, the animal's representation of the environment, its knowledge of the context, is always current. The sensitivity of the mapping system to environmental change is one of its central properties, and it has several implications. For one, it means that stable, or static, elements preferentially enter into, and remain in, the organism's cognitive maps. This gives neural instantiation to the common intuition that static elements are of overriding importance in defining contexts. Second, it involves centrally the spatial mapping system in the detection of surprise, and this might prove to be of importance in understanding certain conditioning phenomena. The main point here, however, is our assertion that an animal 
recognizes a spatial context by retrieving the appropriate internal cognitive map and that this has certain identifiable consequences for its behavior. Any understanding of context effects demands an analysis of cognitive mapping.

\section{Cognitive Mapping}

The cognitive mapping theory of hippocampal function, and the data which support it, have been presented in detail elsewhere (O'Keefe \& Nadel, 1978). For our present purposes, we would like to emphasize three key characteristics of the mapping system as being of particular importance in understanding the nature of context: (1) the redundancy with which cues define places (contexts), and the rapidity with which such place knowledge is acquired; (2) the provision by the map of a steady stream of "predictions" concerning what is to be found, or expected, where; and (3) the recognition of novelty through the incorporation of a match-mismatch system based on the contents of spatial locations. By virtue of these properties, the system provides the basis for spatial context effects in animal learning and takes part in the segmentation of experience into dynamic events and the stable spatial environments containing them. We consider these properties in somewhat greater detail below.

Redundancy. A place (or context) is defined by the set of spatial relations that obtain among a constellation of stimuli. No single cue, or set of cues, is necessary for identification of a place; any cue can be removed from the situation without altering the relations among the remainder. Physiological data in support of this "overdefinition" of places have been reported (O'Keefe \& Conway, 1978), and recent behavioral work confirms this view. Suzuki, Augerinos, and Black (1980) trained rats on a radial arm spatial maze in a controlled cue environment. They found that altering the environment, such as by rotating the maze or cues, failed to disrupt task performance. However, transposing the cues (i.e., altering their spatial relations) did disrupt performance. These data demonstrate that it is the relations among stimuli, and not their mere existence, which define a place. In the same way, a context is always more than the simple sum of the cues present in a situation.

Another point to note about the mapping system is the rapidity with which maps are apparently acquired. Hill (1978) trained rats to obtain food in a shuttlebox and then transferred them to a similar task in a T-maze. He observed that "place" cells in the hippocampus (see O'Keefe \& Nadel, 1978) acquired their fields (the locations in which they fire) within seconds after the rats were introduced into the novel environment. These data suggest that one of the first things an animal learns in any situation is where things are in relation to one another.
Predictions and novelty detection. Maps provide continual "predictions," whose fulfillment or nonfulfillment constitutes the basis for familiarity and novelty, respectively. Once an animal has explored a situation and built a map of it, the animal will be sensitive to subsequent changes in that environment. If something new is introduced, or if familiar things in the situation are rearranged, the animal will react with orienting and exploration, which serve to update the map. Within the hippocampus, this novelty detection function is subserved by "misplace" cells which fire whenever there is a discrepancy between the map's representation of the environment and the actual, experienced environment (see O'Keefe \& Nadel, 1978). There may well be some novelty effects connected with stimuli which have never been experienced anywhere, but most often novelty consists of new arrangements or configurations of familiar things. Given that the hippocampus maps the spatial relations among stimuli, it is uniquely adapted to detect changes in these relations.

\section{Functions of the Mapping System}

The detection of novelty outlined above should be considered a major function of the mapping system. A stimulus introduced into an environment for the first time attracts the animal's attention because there is no representation of that stimulus in the internal map for that situation. Once the animal has incorporated a stimulus into the appropriate map, only subsequent changes in its location, or relevance to some biological need, will attract attention and/ or exploration (see, e.g., Wilz \& Bolton, 1971). Although based on research with static cues, these conclusions also apply to the phasic stimuli employed in classical conditioning paradigms. Shalter (1975, 1978) conducted habituation tests with fowl in the laboratory and in the field and found that the birds' phonoresponses to phasic auditory stimuli depended on the location of the stimulus. Once responsiveness to the stimulus had waned, shifting its location restored the birds' responses. Similarly, Whitlow, Pfautz, and Wagner (cited in Wagner, 1976) showed that the rabbit's long-term habituation decrement to phasic tonal stimuli depends on the place of exposure. Two groups of rabbits were exposed to tones and tested for retention of habituation 2 days later. One group simply sat in their home cages during the interval between exposure and testing, whereas the other group received additional exposure to the test apparatus in the absence of tone presentations. Whitlow et al. found that the rabbits maintained in their home cages during the retention interval showed good retention of habituation, whereas those given additional context-alone experience showed renewed responsiveness to the tone. These context-alone sessions would, from our point of view, cause revision 
of the animals' maps to indicate that the stimulus no longer occurred there. Taken together with results showing that animals with hippocampal damage have normal short-term, but not long-term, decrements in responsiveness to iterated stimuli (cf. O'Keefe \& Nadel, 1978, for documentation), these data critically implicate the mapping system of the hippocampus in mediating the effects of novelty, regardless of whether the stimulus is phasic or static. If there is a difference between phasic and static stimuli in this regard, it may be that phasic stimuli, because of their discontinuous nature, are absorbed into the map less readily than are static stimuli.

The point of interest here is what role this novelty detection plays in behavior. We have earlier stressed the rather direct, observable effect-the elicitation of exploration. Here, we will also discuss the possible role this novelty detection might play in the learning process. We see novelty (surprise) as one of several factors that influence the way in which animals comprehend their environments, and consequently what they learn about them. A novel event (whether CS or US) "stands out" from its context more than does a familiar one by virtue of the fact that it finds no representation in the animal's map of that context and, hence, triggers surprise and draws attention. This means that novel events will more readily enter into associations than will events that have already been absorbed into the context. In the absence of the hippocampal context system and its novelty/familiarity functions, associations between events will depend more heavily on other factors, like simple temporal contiguity.

Another obvious function of the map is to provide information that can be used by the animal to solve its daily problems: where to go to find food when hungry, or water when thirsty, or safety when threatened, and so on, as well as what are the best (safest, shortest) routes to these things in a given context. We have labeled all these uses of map knowledge "place strategies" and contrasted them with behavioral strategies not based on an internal spatial map but, rather, upon internal representations of specific elements in the environment (cue strategies) or upon internal representations of particular behaviors (response strategies). These different, sometimes competing, strategies have qualitatively different properties as well as different neural substrates. Part of the evidence implicating the hippocampus in spatial mapping is the observation that damage to this neural system causes a selective defect in place learning and the use of place strategies, and the loss of exploration from the animal's repertoire (cf. O'Keefe \& Nadel, 1978).

One further point deserves mention here. Although place strategies and novelty detection both depend on an intact spatial mapping system, these two functions are largely independent of one another. That is, the detection of novelty proceeds whether or not the animal's behavior is being controlled by place strategies. While place strategies do, indeed, interact directly with other strategies, often in accordance with a competitive model, novelty detection depends upon another aspect of cognitive mapping - the predictions about what will be contained where are generated from the map. This sense of context as container provides the major reason for rejecting traditional accounts of context.

\section{Context in Conditioning}

Role of novelty detection. Accompanying the decrease in responsiveness to iterated stimuli is the loss in associability of the stimulus known as latent inhibition. Cognitive mapping theory provides a ready explanation for this effect. We have argued that the attention-attracting properties of novel stimuli arise from the fact that they are not represented in the organism's map of the environment and that attention to stimuli wanes as they come to be adequately represented (and hence predictable). When a US is introduced, it is unlikely to be associated with familiar, previously irrelevant, stimuli that have, as it were, faded into the background. We refer to this process as "absorption by the context." In the absence of the mapping system, novelty effects are rendered inoperable, and an animal's responsiveness to a stimulus becomes a function of what we have elsewhere termed its noticeability (O'Keefe \& Nadel, 1978). In line with this view, hippocampal lesions, which we assume disrupt the functions of the mapping system, cause defects in latent inhibition (Solomon \& Moore, 1975). This cannot be considered strong evidence for our position, since purely attentional views of hippocampal function make the same prediction.

More convincing support comes from studies on the factors controlling latent inhibition in normal animals. A number of studies have demonstrated that the context of exposure is an important determinant of latent inhibition. A study by Lubow, Rifkin, and Alek (1976) is particularly instructive in this regard. They preexposed two groups of rats to odors prior to training them on an olfactory discrimination. One group was trained in the preexposure context, whereas the other group was trained in a novel environment. In comparison with controls that received no preexposure, stimulus preexposure retarded learning when training took place in the preexposure environment but facilitated it in a novel environment. These data show that the loss of associability following stimulus preexposure is specific to the environment in which exposure occurred.

Studies by Donegan, Pfautz, and Wagner (cited in Wagner, 1979), using the rabbit eyeblink and CER preparations, also point to the importance of context in mediating latent inhibition. Like the habituation study noted earlier, Donegan et al. found that latent 
inhibition could be "extinguished" by giving the animal context-alone sessions between preexposure and conditioning. The studies just described provide strong support for the notion that exposure effects depend on where the stimulus is exposed. An as yet untested prediction is that shifting the location of the exposed stimulus within the preexposure environment should also attenuate latent inhibition.

Another paradigm in which the novelty detection function of the mapping system may be important is blocking. At present there is no satisfactory account of this centrally important phenomenon. We saw earlier that the explanation of blocking offered by the Rescorla-Wagner model cannot account for the results of studies where the deletion of a US causes unblocking (e.g., Dickinson et al., 1976). Similarly, Mackintosh's account of blocking cannot deal with the results of taste aversion studies where blocking is obtained on the initial compound trial (Batson \& Best, 1979; Willner, 1978) or with the data of Hall and Pearce (1979) discussed above.

Unfortunately, we have no firm evidence on the role of environmental context in blocking. Is blocking context-dependent in the same way as latent inhibition? If so, does damage in the hippocampus disrupt it? The data are presently confusing (Rickert, Bennett, Lane, \& French, 1978; Solomon, 1977; Mackintosh, Note 2). Although there may, indeed, be a defect in blocking after hippocampal disruption, as the latter two studies suggest, it cannot be attributed to some lesion-induced failure in assessing or reacting to changing salience. Mackintosh has shown that lesioned animals demonstrate normal "overshadowing" of one CS by another, more salient CS.

In the present confusion, the following considerations, of a necessarily speculative nature, might be of some interest. In conditioning studies, the animal is faced with the problem of figuring out what goes with what. While 100 years of psychology considered temporal contiguity second only to the deity, blocking shows that closeness in time is not by itself sufficient cause for learning. Spatial contiguity (cf. Rescorla \& Cunningham, 1979) and surprise also seem important, if not sufficient, ingredients in the learning equation. When something new happens, the animal learns what is "close" to it, in time and in space, for these are likely to be causally related to the new event. Temporal and spatial contiguity are both medium and message, turned on by the element of surprise. To the extent that the mapping system is crucial to surprise, it could prove central to the blocking effect.

However, until we have more information on the precise nature of the representations subserved by the mapping system, it would be unnecessarily bold, if not altogether brash, to precisely define the stimulus conditions constituting surprise. We can state with some certainty when surprise must occur, such as when a stimulus is not represented in a map at all, or obviously changes locations, but we cannot specify the lower bounds. That is, we do not know how much change the map will tolerate before it tilts. How fine-grained are its representations? (See Thomas \& Riccio, 1979, for some data that might bear on this issue.) Until we have adequate data on the effects of context shifts on blocking, some resolution of the nature of map representations, and a clearer answer to the question of the hippocampal role in blocking, theoretical leaps become pointless.

Role of place strategies. As we have pointed out elsewhere (O'Keefe, Nadel, \& Willner, 1979), a defining feature of classical conditioning experiments is that the animal cannot influence the course of events. This may rule out the successful use of place strategies as a means of task solution, but it does not mean that animals will fail to be influenced by places. In fact, the rapidity with which animals acquire information about the location of things in the environment makes it quite likely that they will "adopt" place strategies in classical conditioning tasks, at least initially.

What does it mean to say that an animal adopts a place strategy in classical conditioning tasks? Consider a situation in which animals receive unsignaled shocks: They rapidly learn to expect shock in that context (the map "predicts" shock) and behave as though they will be shocked at any time. In short, the beasts are continuously afraid. The critical prediction is that this fear should be specific to the context in which shock was given. Blanchard and Blanchard (1969) have provided data on this point: they gave two groups of rats a single $1.3-\mathrm{mA}$ shock and measured postshock crouching in either the same or a different environment. Significant crouching was observed only in those rats tested in the shock environment; rats tested in the novel environment crouched no more than did unshocked controls. They did, however, crouch when returned to the environment in which they had been shocked. Combined with the observation that hippocampal lesions reduce or eliminate the postshock crouching seen in this task (Blanchard, Blanchard, \& Fial, 1970), these data provide support for the notion that the hippocampus subserves "place fear."

The above indicates that an animal's behavior is largely controlled by place strategies when USs are unsignaled. What happens when these USs are signaled by phasic CSs? We would suggest that behavior initially reflects place strategies even when USs are signaled by CSs. This results from rapid learning about places in conjunction with what appears to be slower learning about the specific features of stimuli per se and their specific relations to other stimuli. With extended training, however, animals show less place "conditioning" and more conditioning to the 
nominal CS. We considered this inverse relationship between context and nominal CS conditioning earlier. We feel that this inverse relationship does not reflect competition between contextual "cues" and nominal CSs for a limited amount of associative strength so much as it reflects competition between place and cue strategies. When the animal's behavior is controlled by place strategies, it will not be dependent upon specific cues. Phasic CSs come to control behavior, to overshadow places, because they provide animals with a more effective strategy: they temporally delimit the occurrence of a US in a given context.

Although we still know relatively little about the factors that control the eventual ascendance of phasic CSs over places in most tasks, the data that do exist underscore the importance of temporal factors in effecting this transition. Odling-Smee (1975b, 1978) has delineated some of these factors controlling the use of place strategies in classical conditioning tasks. CSs come to control behavior only when they provide precise information about US onset (OdlingSmee, 1978), but even this is limited to CS-US onset intervals of between 6 and $10 \mathrm{sec}$. Outside this optimal interval, considerable place conditioning seems to remain. One can only hope that future research will further elucidate the factors and mechanisms that determine strategy utilization. It should be noted that even when there appears to be little conditioning to the context, the context may nevertheless be of considerable importance in the ability of the CS to elicit the CR, as Sheafor (1975) has shown.

One preparation in which place strategies are apparent is in contingency learning (Rescorla, 1969b), in which the contingencies obtaining between CSs, USs, and the context are manipulated. In these studies, conditioning of the nominal CS was seen to be an inverse function of putative "context" conditioning. In fact, the contextual "cues" could be so strongly conditioned that the nominal CS would become an inhibitor. The interpretation we put on such studies is that the delivery of unsignaled USs fosters control of the animal's behavior by place strategies and that, to the extent that the animal's behavior is so controlled, it will not be influenced by phasic cues. The more frequent the unsignaled USs, the stronger the place strategy. We can make two predictions here concerning the effects of removing the context system (by making a hippocampal lesion). First, such damage should reduce or eliminate the place fear seen when aversive USs are used, as in the studies of Dweck and Wagner (1970), Kremer (1974), and Odling-Smee (1975a). Second, hippocampal lesions eliminate the competition between place and cue strategies by rendering the former inoperable. Animals with such lesions will be strongly in the grip of cue strategies. This means that they should show excitatory conditioning of the nominal CS in procedures like the truly random control and that the magnitude of this conditioning will depend solely on the probability of the US in the presence of the CS. These predictions remain to be tested.

In certain types of conditioned inhibition training, such as negative correlation learning and Pavlovian discriminative conditioning, the development of inhibition to the nominal CS depends on the animal's use of place information. For example, in Pavlovian discriminative conditioning (Pavlov, 1927), the animal receives trials in which one stimulus $(A)$ is always reinforced and trials in which a second stimulus (B) is always nonreinforced. Under these conditions, B will become an inhibitor (Rescorla \& LoLordo, 1965). According to Rescorla and Wagner (1972), the context acquires excitatory associative strength on the reinforced trials with $\mathrm{A}$, albeit less than it would have if the USs had been unsignaled. Stimulus B, then, will be nonreinforced in compound with an excitatory stimulus (the context) and will acquire negative associative strength. Thus, the nominal CS becomes an inhibitor because it signals the absence of the US when the animal, by virtue of being in a specific context, expects it (the US). By preventing such contextual predictions, hippocampal lesions should disrupt the US anticipation necessary for the development of inhibition to the nominal CS in this procedure. The available evidence is consistent with this view (Micco \& Schwartz, 1971).

In certain other paradigms, such as Pavlovian conditioned inhibition training, place strategies play little role in the development of inhibition. In this paradigm, trials with a stimulus (A) are always reinforced and are interspersed among trials on which a compound of stimuli A and B is always nonreinforced. B becomes an inhibitor because, in its presence, the US predicted by stimulus $A$ fails to appear. Here, it is a stimulus (A), and not the background, which sets the stage for the development of inhibition by predicting what ultimately fails to appear. We expect no effects of hippocampal lesions when the context is not involved, as in this procedure, and there are data consistent with this view (Solomon, 1977).

\section{Conclusions}

We have argued that the traditional view which treats context as merely another cue is incorrect. In place of this invalid notion, we would substitute a model of context which draws upon data from studies of cognitive mapping and puts emphasis on two separate functions: (1) the role of context maps in novelty/familiarity detection; and (2) place learning and the use of place strategies. Seen in this way, aspects of the context can both compete with and contribute to the learning about nominal CSs. Evidence in partial support of this position comes from several sources: (1) a consideration of the role of context in procedures such as latent inhibition, habit- 
uation, and so on; (2) analysis of the functions of the neural system responsible for cognitive mapping, and held to underlie context; and (3) analysis of the effects of damage to this putative context system on a variety of conditioning procedures. Taken together, these data indicate that contexts are internally represented and that much of what animals learn is referenced to these internal contexts, or maps. But these contexts, like places, are not mere cues, or fixed aggregates of cues, to be treated as an $\mathrm{X}$ in a learning equation. Rather, contexts are overdetermined by the cues they contain, any of which could leap from the background into the foreground when the occasion arises.

The reader may have noticed a passing resemblance between the predictions derived from cognitive map theory and those derivable from Wagner's information processing model (Wagner, 1978, 1979). Although both models emphasize the importance of context in conditioning, the underlying mechanisms they propose are quite different. Wagner's model postulates that stimuli are primed into short-term memory either by recent presentation (self-generated priming) or through associative retrieval from longterm memory (retrieval-generated priming). Primed stimuli are processed less effectively than stimuli that have not been primed. As applied to latent inhibition, Wagner assumes that associations between context cues and CSs are established during preexposure. When the animal is returned to the training environment, the context primes the CS into short-term memory, resulting in less processing than would occur if the CS were novel.

Although we find the information processing approach interesting, we see a number of difficulties with the model. First, given his view of context, the numerous stimuli that comprise the context must surely exceed the capacity of the short-term memory system proposed by Wagner. Second, the context should always be primed, since it is always present, and this should make associations between the context and phasic CSs difficult to establish. Finally, there is no reference in this model to the location of a CS within an environment. The studies by Shalter $(1975,1978)$ point to the importance of this factor in habituation, and we suspect it will also be important in latent inhibition. Thus, despite their surface similarities, there are critical differences between Wagner's view and our own.

The present approach is constrained by both psychological and physiological fact. Currently available data indicate that there is an important role for environmental context in a variety of behavioral situations and that the hippocampus plays a role in mediating these effects. While we would like to know more about the nature of maps, and specifically about the nature of their representations, we know enough to assert that contexts are not the same as "cues" and that only theories of learning which recognize this distinction have any chance of mapping reality.

\section{REFERENCE NOTES}

1. Hinson, R., \& Siegel, S. The mechanism of the UCS preexposure effect. Paper presented at the meeting of the Psychonomic Society, Washington, D.C., 1977.

2. Mackintosh, N. J. Personal communication, 1979.

\section{REFERENCES}

Asratyan, E. A. Compensatory adaptations, reflex activity, and the brain. Oxford: Pergamon Press, 1965.

Baker, A. G., \& Mackintosh, N. J. Excitatory and inhibitory conditioning following uncorrelated presentations of $\mathrm{CS}$ and UCS. Animal Learning \& Behavior, 1977, 5, 315-319.

Batson, J. D., \& Best, P. J. Drug-preexposure effects in flavoraversion learning: Associative interference by conditioned environmental stimuli. Journal of Experimental Psychology: Animal Behavior Processes, 1979, 5, 273-283.

Beritoff, J. S. Neural mechanisms of higher vertebrate behavior. Boston: Little, Brown, 1965.

Blanchard, R. J., \& Blanchard, D. C. Crouching as an index of fear. Journal of Comparative and Physiological Psychology, 1969, 67, 370-375.

Blanchard, R. J., Blanchard, D. C., \& Fial, R. A. Hippocampal lesions in rats and their effect on activity, avoidance and aggression. Journal of Comparative and Physiological Psychology, 1970, 71, 92-102.

Dickinson, A., Hall, G., \& Mackintosh, N. J. Surprise and the attenuation of blocking. Journal of Experimental Psychology: Animal Behavior Processes, 1976, 2, 313-322.

Dweck, C. S., \& Wagner, A. R. Situational cues and correlation between CS and US as determinants of the conditioned emotional response. Psychonomic Science, 1970, 18, 145-147.

Estes, W. K. The statistical approach to learning theory. In S. Koch (Ed.), Psychology: $A$ study of a science (Vol. 2). New York: McGraw-Hill, 1959.

Funk \& Wagnall's standard college dictionary. New York: Funk \& Wagnall, 1963.

Hall, G., \& Pearce, J. M. Latent inhibition of a CS during CS-US pairings. Journal of Experimental Psychology: Animal Behavior Processes, 1979, 5, 31-42.

Hill, A. J. First occurrence of hippocampal spatial firing in a new environment. Experimental Neurology, 1978, 62, 282-297.

Hull, C. L. Principles of behavior. New York: Appleton-CenturyCrofts, 1943.

KAMIN, L. J. Predictability, surprise, attention and conditioning. In B. A. Campbell \& R. M. Church (Eds.), Punishment and aversive behavior. New York: Appleton-Century-Crofts, 1969.

Kimmel, H. D., \& RAY, R. L. Transswitching: Conditioning with tonic and phasic stimuli. Journal of Experimental Psychology: General, 1978, 107, 187-205.

Konorski, J. Integrative activity of the brain: An interdisciplinary approach. Chicago: University of Chicago Press, 1967.

KREMER, E. F. The truly random control procedure: Conditioning to the static cues. Journal of Comparative and Physiological Psychology, 1974, 86, 700-707.

Lubow, R. E. Latent inhibition. Psychological Bulletin, 1973, 79, 398-407.

Lubow, R. E., Rifkin, B., \& Alek, M. The context effect: The relationship between stimulus preexposure and environmental preexposure determines subsequent learning. Journal of Experimental Psychology: Animal Behavior Processes, 1976, 2, 38-47.

Mack intosh, N. J. Stimulus selection: Learning to ignore stimuli that predict no change in reinforcement. In R. A. Hinde \& 
J. Stevenson-Hinde (Eds.), Constraints on learning. London: Academic Press, 1973.

Mackintosh, N. J. A theory of attention: Variations in the associability of stimuli with reinforcement. Psychological Review, 1975, 82, 276-298.

Micco, D. J., \& Schwartz, M. Effects of hippocampal lesions upon the development of Pavlovian internal inhibition in rats. Journal of Comparative and Physiological Psychology, 1971, 76, 371-377.

Odling-Smee, F. J. The role of background stimuli during Pavlovian conditioning. Quarterly Journal of Experimental Psychology, 1975, 27, 201-209. (a)

Odling-Smee, F. J. Background stimuli and the inter-stimulus interval during Pavlovian conditioning. Quarterly Journal of Experimental Psychology, 1975, 27, 387-392. (b)

Oduing-Smee, F. J. The overshadowing of background stimuli by an informative CS in aversive Pavlovian conditioning with rats. Animal Learning \& Behavior, 1978, 6, 43-51.

O'KeEFE, J., \& ConWAy, D. H. Hippocampal place units in the freely-moving rat: Why they fire where they fire. Experimental Brain Research, 1978, 31, 573-590.

O'KeEFE, J., \& NADEL, L. The hippocampus as a cognitive map. Oxford: Clarendon Press, 1978.

O'Keefe, J., NAdel, L., \& Willner, J. Tuning out irrelevancy? Comments on Solomon's temporal mapping view of the hippocampus. Psychological Bulletin, 1979, 86, 1280-1289.

Pavlov, I. P. Conditioned reflexes. London: Oxford University Press, 1927.

RANDich, A., \& LoLordo, V. M. Associative and nonassociative theories of the UCS preexposure phenomenon: Implications for Pavlovian conditioning. Psychological Bulletin, 1979, 86, 523-548.

Rescorla, R. A. Pavlovian conditioning and its proper control procedures. Psychological Review, 1967, 74, 71-80.

RESCORLA, R. A. Probability of shock in the presence and absence of CS in fear conditioning. Journal of Comparative and Physiological Psychology, 1968, 66, 1-5.

RESCORLA, R. A. Conditioned inhibition of fear resulting from negative CS-US contingencies. Journal of Comparative and Physiological Psychology, 1969, 67, 504-509. (a)

Rescorla, R. A. Conditioned inhibition of fear. In W. K. Honig \& N. J. Mackintosh (Eds.), Fundamental issues in associative learning. Halifax: Dalhousie University Press, 1969. (b)

Rescorla, R. A., \& Cunningham, C. L. Spatial contiguity facilitates Pavlovian second-order conditioning. Journal of Experimental Psychology: Animal Behavior Processes, 1979, $5,152-161$.

RESCORla, R. A., \& LoLoRdo, V. M. Inhibition of avoidance behavior. Journal of Comparative and Physiological Psychology, 1965, 59, 406-412.

Rescorla, R. A., \& Wagner, A. R. A theory of Pavlovian conditioning: Variations in the effectiveness of reinforcement and nonreinforcement. In A. H. Black \& W. F. Prokasy (Eds.), Classical conditioning II: Current research and theory. New York: Appleton-Century-Crofts, 1972.

RESTLE, F. Discrimination of cues in mazes: A resolution of the place-vs-response question. Psychological Review, 1957, 64, 217-228.

Rickert, E. J., Bennett, T. L., Lane, P., \& French, J. Hippocampectomy and attenuation of blocking. Behavioral Biology, 1978, 22, 147-160.

Shalter, M. D. Lack of spatial generalization in habituation tests of fowl. Journal of Comparative and Physiological Psychology, 1975, 89, 258-262.
Shalter, M. D. Effect of spatial context on the mobbing reaction of pied flycatchers to a predator model. Animal Behaviour, 1978, 26, 1219-1221.

She AFor, P. J. "Pseudoconditioned" jaw movements of the rabbit reflect associations conditioned to contextual background cues. Journal of Experimental Psychology: Animal Behavior Processes, 1975, 104, 245-260.

Skinner, B. F. The behavior of organisms. New York: AppletonCentury-Crofts, 1938

Solomon, P. R. Role of the hippocampus in blocking and conditioned inhibition of the rabbit's nictitating membrane response. Journal of Comparative and Physiological Psychology, 1977, 91, 407-417.

Solomon, P. R., \& Moore, J. W. Latent inhibition and stimulus generalization of the classically conditioned nictitating membrane response in rabbits (Oryctolagus cuniculus) following dorsal hippocampal ablation. Journal of Comparative and Physiological Psychology, 1975, 89, 1192-1203.

Suzuki, S., Augerinos, G., \& Black, A. H. Stimulus control of spatial behavior on the eight arm maze in rats. Learning and Motivation, 1980 (in press).

Tномаs, D. A., \& Riccio, D. C. Forgetting of a CS attribute in a conditioned suppression paradigm. Animal Learning \& Behavior, 1979, 7, 191-195.

Tolman, E. C. Purposive behavior in animals and men. New York: Century, 1932.

Tolman, E. C. There is more than one kind of learning. Psychological Review, 1949, 56, 144-155.

Tомı, A. Interference with autoshaping by prior context conditioning. Journal of Experimental Psychology: Animal Behavior Processes, 1976, 2, 323-334.

Wagner, A. R. Priming in STM: An information processing mechanism for self-generated or retrieval-generated depression in performance. In T. J. Tighe \& R. N. Leaton (Eds.), Habituation: Perspectives from child development, animal behavior, and neurophysiology. Hillsdale, N.J: Erlbaum, 1978.

WAGNER, A. R. Expectancies and the priming of STM. In S. H. Hulse, H. Fowler, \& W. K. Honig (Eds.), Cognitive processes in animal behavior. Hillsdale, N.J: Erlbaum, 1979.

Wagner, A. R. Habituation and memory. In A. Dickinson \& R. A. Boakes (Eds.), Mechanisms of learning and motivation: A memorial volume for Jerzy Konorski. Hillsdale, N.J: Erlbaum, 1979.

Wagner, A. R., \& Rescorla, R. A. Inhibition in Pavlovian conditioning: Applications of a theory. In R. A. Boakes \& M. S. Halliday (Eds.), Inhibition and learning. London: Academic Press, 1972.

Willner, J. A. Blocking of a taste aversion by prior pairings of exteroceptive stimuli with illness. Learning and Motivation, $1978,9,125-140$.

Wilz, K. J., \& Bolton, R. L. Exploratory behavior in response to the spatial rearrangement of familiar stimuli. Psychonomic Science, 1971, 24, 117-118.

\section{NOTE}

1. This case, in which the US is equally likely to occur in the presence and absence of the CS, has come to be known as the "truly random control procedure," and has been advocated by Rescorla (1967) as the appropriate control procedure for Pavlovian conditioning. 